\title{
ON THE HAUSDORFF MEASURE OF CERTAIN PLANE SET PROJECTIONS
}

ABSTRACT. A characterization of plane sets whose projection have zero Hausdorff measure is given. This is obtained through the study of an angular density introduced first by Marstrand [2].

\section{INTRODUCTION}

This paper deals with the projection of certain class of plane $s$-sets over the real line. The goal is to exhibit conditions, on the projection angle, under which the projection set have Hausdorff measure zero.

Let us explain the main objets of these statements. If $s$ is a positive real number and $E \in \mathbb{R}^{2}$, the Hausdorff $s$-measure $m^{s}(E)$ of $E$ is the number

$$
m^{s}(E)=\sup _{\rho>0} \inf \left\{\Sigma_{i}\left|U_{i}\right|^{s}:\left\{U_{i}\right\} \rho-\operatorname{cover} E\right\},
$$

where $\left\{U_{i}\right\} \rho$ - cover $E$ means that $\rho$ is a real positive number, the union $U_{i} U_{i}$ contains $E$ and the diameter $\left|U_{i}\right|$ of $U_{i}$ is less than $\rho$. Yet, for any $s, \rho>0$ and $E \in \mathbb{R}^{2}$, we define $m_{\rho}^{s}(E)$ as the lower bound of $\Sigma_{i}\left|U_{i}\right|^{s}$ over all possible $\rho-\operatorname{cover}\left\{U_{i}\right\}$ of $E$. It is easy to prove that $m^{s}$ is a outher measure in the sense of Caratheodory (see Royden [1]). A set $E$ is called a $s$-set if it is a measurable set, with respect to the outher measure $m^{s}$, and $m^{S}(E)$ is a positive finite number. In addition, if $\theta \in[0,2 \pi)$ and $d, \delta>0$ and $x \in \mathbb{R}^{2}, R(x, \theta, \delta, d)$ denotes the rectangle in $\mathbb{R}^{2}$ with center at $x$, base lenght $2 \delta$, size lenght $2 d$, pointing toward the $\theta$ direction. Also, for $s, q>0, \theta \in[0,2 \pi), E \in I R^{2}$ and $x \in E$ we define the $(\theta, q, s)-$ density of $E$ at $x$ as the limit

$$
\lim _{\rho \rightarrow 0^{+}} \liminf _{\delta \rightarrow 0^{+}} \delta^{-q} m^{s}(E \cap R(x, \theta, \delta, d))
$$


Sometimes, we denote this density by $D_{\theta}^{q, s}(E, x)$. This notion was introduced by Marstrand [2], where it was proved that the $(\theta, 1, s)$ - density of $E$ at almost every point $x \in E$ is infinity when $\theta \in[0,2 \pi), s>0, E$ is a $s$-set and the projection of $E$ along the direction $\theta$ has Lebesgue measure equal zero (see Lemma 12 of Marstrand [2]). If $\theta \in[0,2 \pi)$ and $E \in \mathbb{I}^{2}$, then the projection of $E$ along the direction $\theta$ over any orthogonal direction is denoted by $P(\theta, E)$. Our main theorem is a sort of converse of the Marstrand's Lemma above mentioned.

Theorem 1.- Let $s, q$ be two real positive numbers and $\theta \in[0,2 \pi)$. Suppose that $E \in I R^{2}$ is a s-set for which its $(\theta, q, s)$-density at every point $x \in E$ is infinity. Then $m^{q}(P(\theta, E))=0$.

It is clear that Theorem 1 and the Marstrand's result characterize, up to some restrictions, the directions $\theta$ for which a given fixed $s$-set $E$ projects into Hausdorff $s$ measure zero set. This observation applies to the problem of determinate the set of $\lambda \in R$ so that the difference set $K-\lambda K^{\prime}$, of two fixed real line cantor sets $K, K^{\prime}$, has zero Lebesgue measure. Indeed, [2] proved that such a set of $\lambda$ 's has zero Lebesgue if $E=K \times K^{\prime}$ is a $s$-set for some $s>1$. See [3] for a relation between the above problem and the global study of certain ordinary differential equations.

\section{THE PROOFS}

We start with a classical result due to Vitali which can be found in the book of Falconer [4]. Let $(X, d)$ be a metric space and $\mu$ an outher measure in $X$. We say that a collection $V$ of subsets of $X$ is a Vitali's collection of a given set $E \in X$ if every $U \in V$ is a closed subset of $X$ and for each $x \in E$ and $\varepsilon>0$ there exists a $U \in V$ for which $x \in U$ and $0<|U|<\varepsilon$, where $|U|$ means the diameter of $U$ in $(X, d)$. Now, the metric space $(X, d)$ is a $(\mu, s)$-Vitali space if for each $E \in X$ and any Vitali's collection $V$ of $E$ there exists a countable disjoint subcollection $\left\{U_{i}\right\}_{i \in I N} \subset V$ such that at least one of the following assertions hold

(1) $\sum_{i \in I N}\left|U_{i}\right|^{s}=\infty$

(2) $\mu\left(E \backslash \bigcup_{i \in I N} U_{i}\right)=0$

For a proof of the following Theorem we refer to Falconer [4]. 
Theorem 2.1.- For all $n \in N \mathbb{R}^{n}$, endowed with the usual metric, is a $\left(m^{s}, s\right)$-Vitali space for all $s>0$.

We keep the case $n=1$ as the following immediatly Corollary which is usually called the Vitali's covering Theorem.

Corollary 2.2.- If $E \in R$ and $V$ is a Vitali's collection of $E$ formed by closed intervals, then it is possible to find a countable disjoint subcover $\left\{U_{i}\right\}_{i \in I N} \subset V$ for which either $\sum_{i \in I N}\left|U_{i}\right|=\infty$ or $m^{*}\left(E \backslash \bigcup_{i \in I N} U_{i}\right)=0$.

Here, $m^{*}$ denotes the classical outher measure in $R$ generated by the intervals (see the book of Royden [1]).

Proof of Theorem 1.- Take $\rho>0$ and $M \in(0, \infty)$. Let $V$ be the following collection:

$$
V=\left\{P(\theta, R(x, \theta, \delta, d)): x \in E, 2 \delta \leq \rho \text { and } m^{s}(E \cup R(x, \theta, \delta, d))>M \delta^{q}\right\}
$$

We shall prove that $V$ is a Vitali's collection of $P(\theta, E)$ for which (2) above holds with $\mu=m^{s}$. In fact, take $p=P(\theta, x) \in P(\theta, E)$, then using $D_{\theta}^{q, s}(E, x)=\infty$ we have that for any $d>0$ there exists $\Omega_{d} \in(0, d)$ such that if $0<\delta<\Omega_{d}$ then $m^{s}(E \cap R(x, \theta, \delta, d))>M \delta^{q}$. Let $\varepsilon$ be a positive number and choose any $d>0$. Let us take $\delta$ in the interval $\left(0, \min \left(\Omega_{d}, \rho / 2, \varepsilon / 2\right)\right)$. Then the interval $I=[p-\delta, p+\delta]=P(\theta, R(x, \theta, \delta, d))$ belongs to $V$ and $0<|I|<2 \delta<\varepsilon$. This implies that $V$ is a Vitali's collection of $P(\theta, E)$ formed by closed intervals. Now if $\left\{U_{i}\right\}$ is any countable disjoint subcollection of $V$ we have.

$$
\sum_{i \epsilon}|P(\theta, R(x, \theta, \delta, d))|^{q}=2^{q} \sum_{i} \delta_{i}^{q}<2^{q} M^{-1} m^{s}(E)<\infty
$$

because of $\left\{P\left(\theta, R\left(x_{i}, \theta, \delta_{i}, d_{i}\right)\right)\right\}$ disjoint implies $\left\{R\left(x_{i}, \theta, \delta_{i}, d_{i}\right)\right\}$ disjoint. Therefore we have that

$$
m^{q}\left(P(\theta, E) \backslash \bigcup_{i} P\left(\theta, R\left(x_{i}, \theta, \delta_{i}, d_{i}\right)\right)\right)=0
$$

for some disjoint countable subcollection $\left\{U_{i}\right\}$ of $V$ because of Theorem 2.1. So if $\rho>0$ we have $m_{\rho}^{q}\left(P(\theta, E) \backslash \bigcup_{i} P\left(\theta, R\left(x_{i}, \theta, \delta_{i}, d_{i}\right)\right)\right)+m_{\rho}^{q}\left(P(\theta, E) \cap\left[\bigcup_{i} P\left(\theta, R\left(x_{i}, \theta, \delta_{i}, d_{i}\right)\right)\right]\right) \leq 2^{q} m^{s}(E) M^{-1}$ then we have

$$
m_{\rho}^{q}(P(\theta, E)) \leq 2^{q} m^{s}(E) M^{-1}
$$

and thus we are done because $\rho$ and $M$ are arbitraries. The proof is completed. Now we state some corollaries of Theorem 1 . 
Corollary 2.3.- Let $s, q$ be positive numbers, $E \subset I^{2}$ and $\theta \in[0,2 \pi)$. Assume that $q \geq s$. If the $(\theta, q, s)-$ density of $E$ at almost every point $x \in E$, with respect to the outher measure $m^{s}$, is infinity then $m^{q}(P(\theta, E))=0$.

Proof.- Let $F$ be defined as $F=\left\{x \in E: D_{\theta}^{q, s}(E, x)=\infty\right\}$. Then using $m^{s}(F \cap R(x, \theta, \delta, d))=m^{s}(E \cap R(x, \theta, \delta, d))$ (which is true because of $m^{s}(E \backslash F)=0$ ) we have $D_{\theta}^{q, s}(E, x)=\infty$ for all $x \in F$. Therefore

$$
m^{q}(P(\theta, E)) \leq m^{q}(P(\theta, E \backslash F)) \leq m^{q}(E \backslash F)=0
$$

because of $q \geq s$ (see Falconer [4]). The proof is completed.

Corollary 2.4.- Let $E$ be a 1 -set in $\mathbb{R}^{2}$ and $\theta \in[0,2 \pi) . D_{\theta}^{1,1}(E, x)=\infty$ for almost every $x \in E$, with respect to the Lebesgue real measure $m$ if and only if $m(P(\theta, E))=0$.

Proposition 2.5.- If $E$ is a $s$-set, $s \geq 0, \theta \in[0,2 \pi)$ and the $(\theta, 1, s)$-density of each point $x \in E$ is infinity then $m(P(\theta, E))=0$.

This proposition follows from the proof of the Theorem 1 and Corollary 2.2.

A simple example is the following. Let $E$ be the segment $\{0\} \times[1,2]$ in $I R^{2}$. Using that $E$ is a rectificable curve we have that $m^{1}(E)=$ lengh of $E$ (see Falconer [4]). Therefore, $E$ is a $1-$ set of $I R^{2}$. An easy computation gives $D_{\theta}^{1,1}(E, x)=1 / 2 \cos \theta$ for all $x \in E$ and $\theta \neq \pi / 2$. Using our results, we obtain $m(P(\theta, E))>0$ if different from $\pi / 2$. This last affirmation can be easily checked using the picture.

\section{REFERENCES}

1. ROYDEN H.L., Real analysis, Macmillan Company, New York.

2. MARSTRAND J.M., Some fundamental geometrical properties of plane sets of fractional dimensions, Proceedings of the London Math. Soc. (3), 4, (1954) (2).257-302.

3. PALIS J. - TAKENS F., Hyperbolicity and sensitive chaotic dynamics at homoclinic bifurcations, Cambridge University Press (35), (1993).

4. FALCONER K.J., The geometry of fractal sets, Cambridge University Press (85). 